\title{
Ist Facebook schlecht für die psychische Gesundheit?
}

Brailovskaia J et al. Comparing mental health of

Facebook users and Facebook non-users in an inpatient sample in Germany. J Affect Disord

2019. doi: 10.1016/j.jad.2019.08.078

Soziale Medien wie das Portal Facebook sind aus der heutigen Zeit kaum noch wegzudenken und werden auch von Patienten mit psychischen Erkrankungen genutzt. Brailovskaia und Kollegen wollten wissen, ob sich die Nutzung und die tägliche Nutzungsdauer von Facebook auf die psychische Gesundheit auswirken und haben dazu eine Studie mit stationären Patienten einer deutschen psychosomatischen Rehaklinik durchgeführt.

Eine Welt ohne soziale Medien und Netzwerke wie Facebook ist für viele Menschen heute nicht mehr vorstellbar. Nach aktuellen Schätzungen zählt Facebook heute mehr als 2,3 Billionen Mitglieder, 1,5 Billionen von ihnen sind täglich auf Facebook online. Da sich die Mitglieder u. a. in Gruppen austauschen und dadurch auch Feedback und soziale Unterstützung bekommen, vermuten Wissenschaftler heute positive Effekte auf die mentale Gesundheit. Da es auf der anderen Seite aber auch schon Studien gibt, die eher das Gegenteil vermuten lassen, wollten Brailovskaia und ihre Kollegen hier einmal genauer nachschauen und haben eine Studie zum Thema durchgeführt.

Ihre Stichprobe umfasste dabei Patienten einer psychosomatischen Rehabilitationsklinik in Deutschland, die dort primär aufgrund von affektiven Störungen oder Angststörungen behandelt wurden. Um die mentale Gesundheit objektivieren zu können, griffen die Forscher auf das 2-Faktoren-Modell der mentalen Gesundheit von Keyes zurück. Das Modell unterscheidet dabei die Faktoren der positiven mentalen Gesundheit und der negativen mentalen Gesundheit. Die Forscher wollten nun wissen, ob sich in der Patientengruppe die Mitgliedschaft auf Facebook auf die mentale Gesundheit auswirkt, und ob dabei auch die Nutzungsdauer eine Rolle spielen könnte.

Im Zeitraum zwischen Juni 2018 und November 2018 füllte jeder neue Patient der Klinik kurz nach der Aufnahme am Computer einen Fragebogen aus. Dieser bestand aus Skalen zur Beurteilung der positiven mentalen Gesundheit und zur negativen mentalen Gesundheit und fragte zudem nach Nutzung und ggf. Nutzungsdauer von sozialen Netzwerken, im Speziellen von Facebook. Schließlich bildeten die Studienautoren 2 Gruppen: die FacebookNutzer und die Facebook-Nicht-Nutzer. Diese beiden Gruppen wurden in Hinblick auf die mentale Gesundheit miteinander verglichen.

\section{Schlaflosigkeit bei Facebook- Nutzern}

Im Studienzeitraum nutzten 336 Klinikpatienten Facebook, 265 Patienten bildeten die Gruppe der Nicht-Nutzer. In Hinblick auf die Indikation zur stationären Therapie konnten die Autoren keine bedeutsamen Gruppenunterschiede feststellen. Ins- gesamt verbrachten 17,3\% der FacebookNutzer mehr als 1 Stunde täglich auf dem Portal. Eine Facebook-Nutzung korrelierte deutlich mit den Werten für eine negative mentale Gesundheit wie somatoformen Symptomen, Depressionen, sozialem Stress und Schlaflosigkeit.

Der Unterschied beider Gruppen war dabei signifikant. Darüber hinaus konnten die Forscher feststellen, dass eine längere Nutzungsdauer mit einem höheren Risiko für eine starke Ausprägung der Skalen für die negative mentale Gesundheit einherging. Die Autoren halten ihre Ergebnisse für therapierelevant und empfehlen daher dringend, die Facebook-Nutzung zu Beginn einer stationären psychosomatischen Therapie anzusprechen und auch in Hinblick auf das Behandlungsergebnis mit zu berücksichtigen.

In dieser klinischen Studie zeigten Patienten einer psychosomatischen Rehaklinik, die Facebook nutzten, im Vergleich zu Patienten, die Facebook nicht nutzten, ein höheres Risiko für eine negative mentale Gesundheit und Symptome wie Schlafstörungen und sozialen Stress. Da die negativen Effekte zudem positiv mit der Nutzungsdauer korrelierten, empfehlen die AutorInnen ausdrücklich, eine etwaige Facebook-Nutzung bei der Therapieplanung zu thematisieren.

Dipl.-Psych. Annika Simon, Hannover 